\title{
Sudden-Onset Paraplegia and Ischemia of the Lower Extremities from Acute Aortic Occlusion following Type A Acute Dissection
}

\author{
Lei Yu, Tianxiang Gu*, Enyi Shi and Qin Fang
}

Department of Cardiac Surgery, China Medical University, Shenyang, China

\begin{abstract}
Herein, we report the case of a 51-year-old man who presented with sudden-onset paraplegia and ischemia of lower extremities, with low back pain. CT scanning revealed Type A acute dissection with occlusion of the infrarenal abdominal aorta and bilateral iliac arteries. A lumbar catheter was inserted for cerebrospinal fluid drainage and we repaired Type A aortic dissection with ascending aorta and total arch replacement combined with stent-graft elephant trunk technique by using innominate cannulation and an aorto-femoral bypass. On postoperative day three, ischaemic symptoms including paraplegia totally disappeared and he was discharged from the hospital on postoperative day 44 .
\end{abstract}

\section{Introduction}

Acute aortic occlusion is a rare event in patients with known history of peripheral vascular disease, and is characterized by devastating outcomes, with extremely high morbidity and mortality rates [1]. Its possible causes include in situ thrombosis, aortic saddle embolus, as well as an intimal flap resulting in dissection and thrombosis [2]. The diagnosis can occasionally be mistaken for stroke or a central nervous system lesion $[2,3]$. Here, we present the unusual case of a patient who presented with sudden-onset paraplegia and ischemia of the lower extremities from acute aortic occlusion following Type A acute dissection.

\section{Case Report}

A 56-year-old man was admitted to the emergency department, complaining of sudden onset of low back pain and both lower limbs, which were accompanied by lower limb cyanosis and weakness bilaterally, approximately 4 hours before admittance. Physical examination revealed sensory loss with severe cyanosis of bilateral lower limbs with no pulse of the femoral artery or its distal arteries. The sensory deficit rapidly progressed to full paraplegia. His renal function was normal with myoglobinuria. Computed tomography scanning revealed Type A acute dissection with occlusion of the infrarenal abdominal aorta and bilateral iliac (including common, external, and internal iliac) arteries with thrombus formation (Figure 1). The patient underwent emergent surgical treatment. A lumbar catheter was inserted for cerebrospinal fluid drainage, with a cerebrospinal fluid opening pressure of $16 \mathrm{mmHg}$ and intradural pressure maintained at $10 \mathrm{mmHg}$. We repaired Type A aortic dissection with ascending aorta and total arch replacement combined with stent-graft elephant trunk technique by using innominate cannulation. The procedure was carried out by a median sternotomy and total cardiopulmonary bypass with selective cerebral perfusion. Supracommissural aortic replacement was performed with a straight $28 \mathrm{~mm}$ prosthetic graft (Hemashield Platinum, MAQUET, Cardiovascular LLC, Wayne NJ, CA, USA), since the root was not involved in the dissection and no aortic insufficiency was present. A self-expandable 28-mm stent graft (MicroPort Medical Co, Ltd, Shang hai, China) and a 4-branch prosthetic graft (Hemashield Platinum, MAQUET, Cardiovascular LLC, Wayne NJ, USA) were used in total arch replacement combined with stent-graft elephant trunk implantation. After CPB, the patient's right lower-extremity perfusion improved, and the distal pulses returned, however, both the femoral pulse and vascular Doppler flow signal of the left leg were not restored. Finally, an aorto-femoral bypass was performed using an 8-mm Gore-Tex Vascular Graft (W.L. Gore \& Associates, Inc., Flagstaff, AZ, USA) in an end-to-end fashion to 1 limb of the 4-branch prosthetic graft (Figure 2). However during the operation we did not find the thrombus formation in the femoral artery. The patient's left lower-extremity perfusion improved, and the distal pulses returned after the procedure. Post-operative treatment for spinal cord injuries and ischemia-reperfusion injuries included that increasing the blood pressure $>140 \mathrm{mmHg}$, intravenous methylprednisolone 1 gram/day for 3 days, and maintaining the cerebrospinal fluid pressure $<10 \mathrm{~cm}$ $\mathrm{H}_{2} \mathrm{O}$. On postoperative day one, continuous hemodiafilteration was started because of acute renal failure (ARF) and reduced urinary output. On postoperative day three, ischaemic symptoms including paraplegia totally disappeared. The patient's renal function was rescued from dialysis 1 month later and he was discharged from the hospital on postoperative day 44. Postoperative 3-D reconstruction of the CT-scan revealed the vascular prosthesis of the ascending aorta, the 4-branch prosthetic graft, the stent graft in the descending aorta and the aortofemoral graft were all patent and no thromboemboli were identified (Figure 3).

\section{Discussion}

Acute aortic occlusion carries a very poor prognosis unless it is treated immediately, so it is very important to differentiate this condition from other spinal vascular pathologic phenomena that produce paraplegia. Virtually all cases can be adequately diagnosed by contrast CT, as in the presented case. Not only does the CT allow a correct diagnosis, but it also allows surgical planning and evaluation of possible collateral circulation. Although spinal cord ischemia on

*Corresponding author: Tianxiang Gu, Department of cardiac surgery, The first affiliated hospital, China Medical University, Shenyang, P.R. China 110001, Tel: 86-24-83283455; E-mail: cmugtx@sina.com

Received October 01, 2015; Accepted October 20, 2015; Published October 26, 2015

Citation: Yu L, Gu T, Shi E, Fang Q (2015) Sudden-Onset Paraplegia and Ischemia of the Lower Extremities from Acute Aortic Occlusion following Type A Acute Dissection. Overview Data. Cardiol Pharmacol S1: 004. doi:10.4172/23296607.1000004

Copyright: ( 2015 Yu L, et al. This is an open-access article distributed under the terms of the Creative Commons Attribution License, which permits unrestricted use, distribution, and reproduction in any medium, provided the original author and source are credited. 
Citation: Yu L, Gu T, Shi E, Fang Q (2015) Sudden-Onset Paraplegia and Ischemia of the Lower Extremities from Acute Aortic Occlusion following Type A Acute Dissection. Overview Data. Cardiol Pharmacol S1: 004. doi:10.4172/2329-6607.1000004

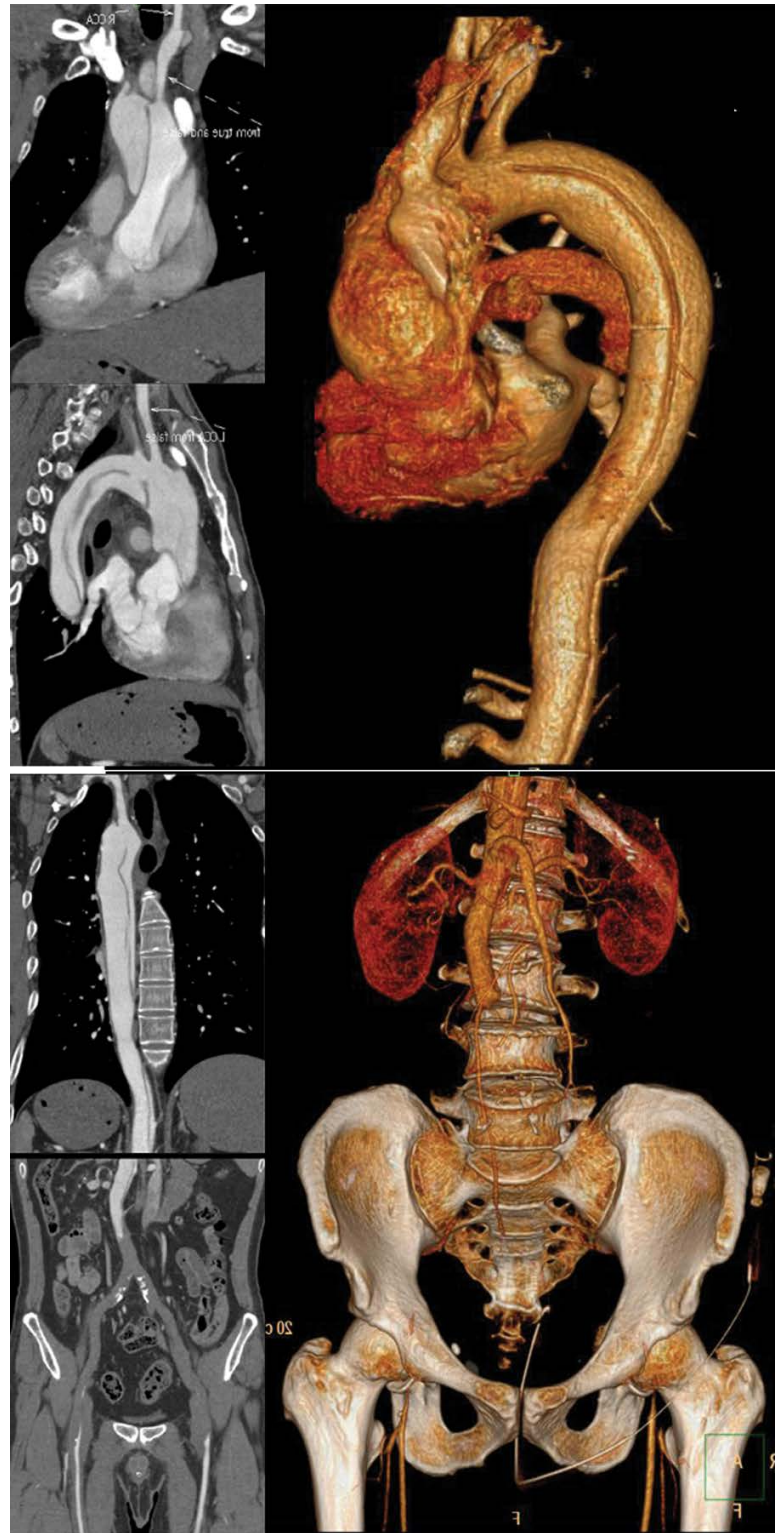

Figure 1: Computed tomography scanning revealed acute aortic occlusion (arrow) following Type A acute dissection.

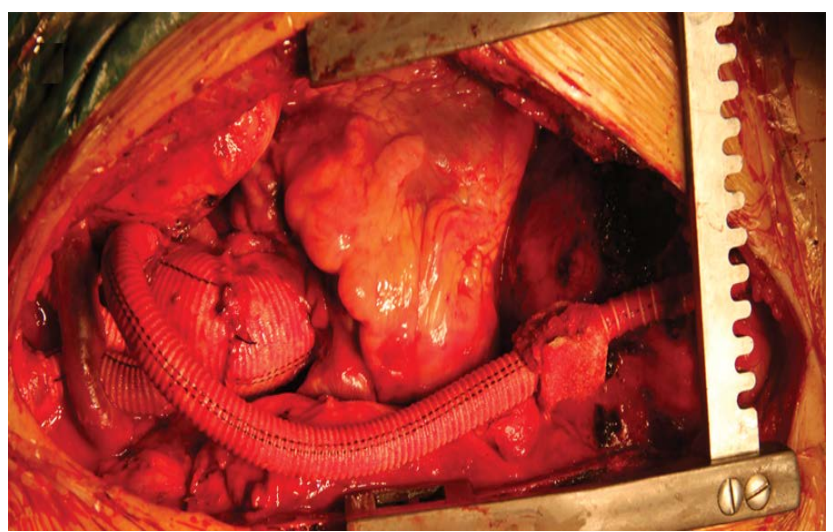

Figure 2: Introperative imaging.

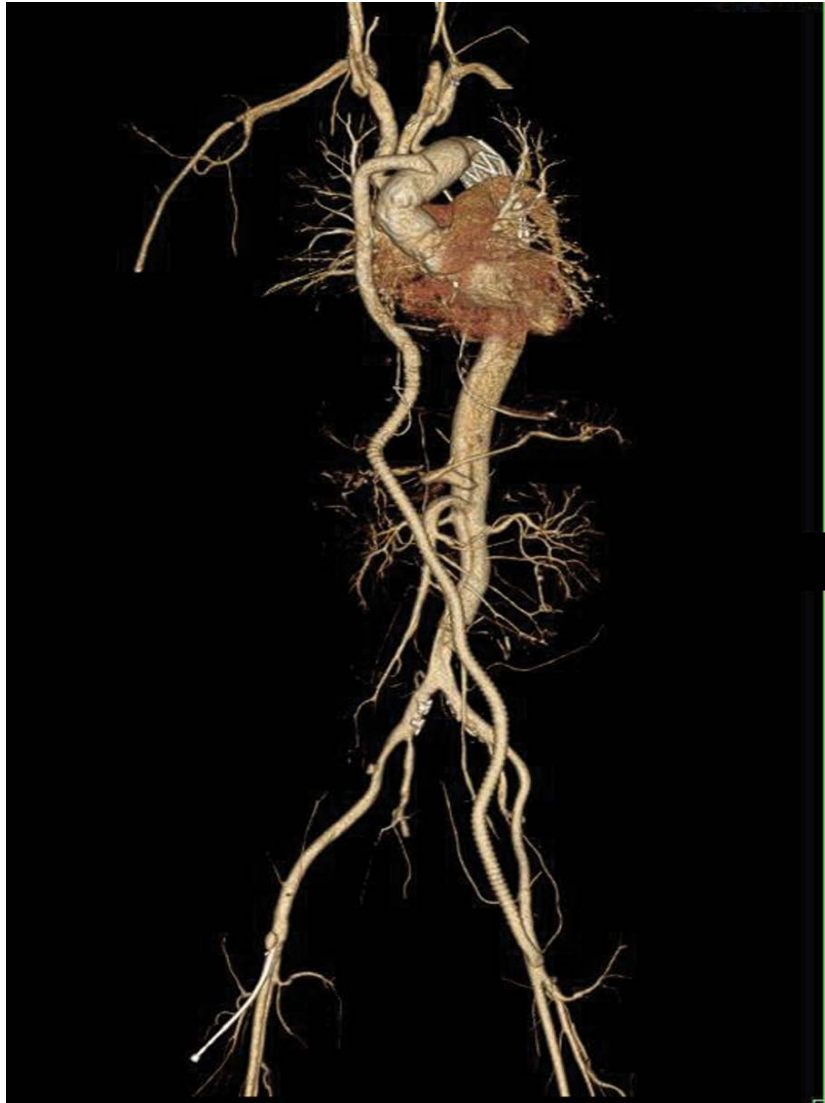

Figure 3: Postoperative 3-D reconstruction of the CT-scan revealed the vascular prosthesis of the ascending aorta, the 4-branch prosthetic graft, the stent graft in the descending aorta and the aortofemoral graft were all patent.

the basis of aortic dissection is considered rare [4], the clinical and imaging findings of our case further point out that once the condition is suspected, time is a critical factor and radiologists, neurologists as well as internal specialists should be vigilant for this emergency.

Neurological disorders after aortic dissection are not uncommon, and paraplegia is one manifestation, due to either a spontaneous or iatrogenic compromise of spinal blood supply [5]. If symptoms are not rapidly regressive, cerebrospinal fluid drainage is certainly indicated and may have beneficial effects [5].There have been some case reports of successful reversal of delayed-onset paraplegia using cerebrospinal fluid drainage after surgical treatment of Type A acute dissection [6]; however, there are few reports of recovery from short time-onset paraplegia. In this report, we described a case of immediate postoperative spinal cord damage that was reversed by cerebrospinal fluid drainage on postoperative day three. In our opinion, the value of cerebrospinal fluid drainage in the resolution of paraplegia is directly related to its implementation before irreversible ischemic damage occurs. The prompt cerebrospinal fluid drainage decreased the cerebrospinal fluid pressure, and the increased spinal perfusion through the collateral circulation prevented irreversible ischemic changes of the spinal cord.

Clinically, ischemia of the lower extremities is most commonly recognized when bilateral or unilateral femoral pulses are lost. Bossone reported that one third of patients with Type A dissection had pulse deficits and that $29 \%$ of those patients had limb ischemia [7]. In a 
patient with aortic dissection and lower extremity ischemia, one option is to repair the aorta to re-establish flow within the true lumen and restore flow to the lower extremities. However, if this technique is unavailable or unsuccessful, open procedures should be performed. In our patient, after repair of Type A aortic dissection the rapid restoration of flow into the true lumen had improved perfusion of the spinal cord and diminished the right lower-limb ischemia. However, the patient's left lower-extremity perfusion was not improved, so we performed an aorto-femoral bypass. Although the CT scan before surgery showed embolism of abdominal artery (below the level of renal arteries) and iliac arteries. However, during the surgery we did not find the embolism in the femoral artery. The most likely reason was that the distal blood could not be supplied because of the false lumen instead of thrombosis, so the patient did not receive anticoagulant therapy after the surgery and no residual thromboemboli were identified by the postoperative CT scan.

Paraplegia is caused by occlusion of spinal arteries and occlusion of both renal arteries due to dissection or thrombosis can cause ARF [8]. Aortic occlusion can be complicated by rhabdomyolysis, as in this case, although the renal artery were patient, contributing to ARF. Haemodialysis and continuous venovenous haemodiafiltration (CVVHDF) are well-established treatment methods in patients with acute kidney injury. It is an efficient method in removing solutes over the course of 24 to 48 hours, just as conventional haemodialysis [9]. The CVVHDF eliminates pro-inflammatory substances, free radicals, endotoxins and cytokines, and this seems to be particularly beneficial in septic patients [10]. Our patient received CVVHDF and his renal function was rescued from dialysis 1 month later. This demonstrated that CVVHDF was important for the patient to depress the risk of further renal insufficiency.
In conclusion, we report a case of acute aortic occlusion following Type A acute dissection that presented as sudden-onset paraplegia and ischemia of the lower extremities. The patient fully recovered after emergent surgery and had no neurologic deficits. Although acute aortic occlusion following Type A acute dissection is rare, it should be considered in the differential diagnosis of patients who present emergently with acute-onset paraplegia and pulseless femoral arteries bilaterally. Prompt diagnosis and intervention can prevent morbidity and death.

\section{References}

1. Triantafyllopoulos GK, Athanassacopoulos M, Maltezos C, Pneumaticos SG (2011) Acute infrarenal aortic thrombosis presenting with flaccid paraplegia. Spine (Phila Pa 1976) 36: E1042-1045.

2. Babu SC, Shah PM, Nitahara J (1995) Acute aortic occlusion--factors that influence outcome. J Vasc Surg 21: 567-572.

3. Surowiec SM, Isiklar H, Sreeram S, Weiss VJ, Lumsden AB (1998) Acute occlusion of the abdominal aorta. Am J Surg 176: 193-197.

4. Gaul C, Dietrich W, Friedrich I, Sirch J, Erbguth FJ (2007) Neurological symptoms in type A aortic dissections. Stroke 38: 292-297.

5. Sinha AC, Cheung AT (2010) Spinal cord protection and thoracic aortic surgery. Curr Opin Anaesthesiol 23: 95-102.

6. Girdauskas E, Kuntze T, Walther T, Mohr FW (2008) Delayed paraplegia associated with vertebral necrosis after type A dissection surgery. Eur J Cardiothorac Surg 33: 121-123.

7. Bossone E, Evangelista A, Isselbacher E, Trimarchi S, Hutchison S, et al. (2007) Prognostic role of transesophageal echocardiography in acute type A aortic dissection. Am Heart J 153: 1013-1020.

8. Sampathkumar K, Sooraj YS, Ajeshkumar RP, Mahaldar AR, Muthiah R (2007) The Case | Acute paraplegia with anuric ARF. Occlusive aortic thrombus with ischemia of spinal cord and kidneys. Kidney Int 72: 657-659.

9. Konopka A, Banaszewski M, Wojtkowska I (2011) Early implementation of continuous venovenous haemodiafiltration improves outcome in patients with heart failure complicated by acute kidney injury. Kardiol Pol 69: 891-896.

10. Morgera S, Slowinski T, Melzer C, Sobottke V, Vargas-Hein O, et al. (2004) Renal replacement therapy with high-cutoff hemofilters: Impact of convection and diffusion on cytokine clearances and protein status. Am J Kidney Dis 43: 444-453.
This article was originally published in a special issue, Management of Peripheral Arterial Disease handled by Editor. Dr. Smriti. James Cook University, USA 